\title{
Orexin-A Attenuates Inflammatory Responses in Lipopolysaccharide-Induced Neural Stem Cells by Regulating NF-KB and Phosphorylation of MAPK/ P38/Erk Pathways
}

\author{
Wen Ye' \\ Yan $\mathrm{Yan}^{2}$ \\ Yunliang Tang' \\ Xiaoyang Dong' \\ Gengfa Chen' \\ Junwei Kang' \\ Lianghua Huang' \\ Qi Xiong' \\ Zhen Feng'
}

'Department of Rehabilitation, The First Affiliated Hospital of Nanchang

University, Nanchang City, Jiangxi

Province, People's Republic of China;

${ }^{2}$ Department of Nephrology, The First

Affiliated Hospital of Nanchang

University, Nanchang City, Jiangxi

Province, People's Republic of China
Correspondence: Zhen Feng

Department of Rehabilitation, The First

Affiliated Hospital of Nanchang

University, Nanchang City, Jiangxi

Province, People's Republic of China

Email medstu@I26.com
Background: Neuronal damage is the main cause of neurological diseases. Neural stem cells (NSCs) have the functions of cell repair and replacement of neurons, secretion of neurotrophic factors, and immune regulation of the neural microenvironment.

Objective: Previous study found that Orexin-A had a protective effect on neurons in the central nervous system, but it is lacking in making great efforts on the function of Orexin-A on NSCs. This study aimed to investigate the anti-inflammatory responses and signaling mechanisms of Orexin-A on lipopolysaccharide (LPS)-induced NSCs.

Methods: Quantitative real-time polymerase chain reaction was used to detect the mRNA level. Signaling pathway-related protein expression was detected by Western blot. The proliferation and migration of NSCs were investigated by Cell Counting Kit-8 (CCK-8) detection kit and transwell assay. Besides, the staining of hematoxylin and eosin (HE) was performed to study the morphology of cell.

Results: Orexin-A decreased the pro-inflammatory cytokines of IL-1 $\beta$, TNF- $\alpha$, and IL-6 induced by LPS by regulating nuclear factor-k-gene binding (NF-kB) and phosphorylation of P38/Erk-mitogen-activated protein kinases (MAPKs) pathways, but not p-JNK signaling.

Conclusion: Our findings indicate that Orexin-A can alleviate the inflammatory response of NSC. It can provide beneficial help in neural stem cell therapy applications.

Keywords: NSCs, Orexin-A, NF-kB, MAPK

\section{Introduction}

Neurological diseases (NDs) are a major public health problem, with high prevalence, and leading to disability and mortality. ${ }^{1-3}$ The World Health Organization estimates that ND and its sequelae affect as many as one billion people worldwide. Cerebrovascular diseases, ${ }^{4}$ neurodegenerative diseases, ${ }^{5}$ and mental illnesses such as stroke ${ }^{6}$ and dementia are the main causes of death and disability. ${ }^{7}$ The essence of neurological diseases is neuronal damage. ${ }^{8,9}$ Primary injury is directly caused by an external force, The mechanism of secondary injury is very complicated, including excitotoxicity, oxidative stress, intracellular calcium overload, inflammatory response, and cell apoptosis. ${ }^{10}$ However, adult mammals, including humans, have very limited brain regeneration capabilities. ${ }^{11}$ There is a lack of clinically effective treatments for diseases in which neuron loss leads to the destruction of neural connections and impaired nerve function. Meanwhile, the self-renewal and multi- 
potential abilities of neural stem cells are important for the recovery of the injured central nervous system (CNS) in the brain. ${ }^{12,13}$ Transplanting neural stem cells into the brain to replace the function of the lost nerve cells in the brain is one of the potential treatment measures. It is also important to understand the interaction between NSC and immunity to prevent disease progression.

As we all know, Lipopolysaccharide (LPS) is the main component of the outer membrane of gram-negative bacteria. ${ }^{14}$ It is composed of lipid A, core polysaccharide, and O-specific chain. LPS induces Neural stem cells (NSCs) to construct in vitro inflammation model. ${ }^{15}$ The Toll-like receptor 4 (TLR4) receptor can recognize LPS, transmit signals into the cell and activate the intracellular signal transduction pathway. ${ }^{16,17}$ After activating Myeloid differentiation factor 88 (MyD88) and IL-1 receptorassociated kinase (IRAK), the MyD88 and MyD88 transfer protein analogs aggregate into the receptor complex to activate IRAK phosphorylation. ${ }^{18,19}$ Then IRAK dissociates from the complex, transmits the signal to TRAF6, and activates it. ${ }^{20}$ Activated TNF receptor-associated factor 6 (TRAF6) conducts signal transduction by activating NF$\mathrm{kB}$-inducing kinase (NIK) and Transforming growth factor beta-activated kinase 1 (TAK1) and activates the corresponding NF-kB and mitogen-activated protein kinase (MAPK) pathways. $^{21}$ Eventually, it causes the release of IL-1, IL-6, TNF- $\alpha$, and NO to form an inflammatory response. However, the role of NF-kB and MAPK in the proliferation and differentiation of NSCs is still debatable.

Orexin-A is involved in a variety of central and peripheral regulatory processes, which is a 33 amino acid excitatory neuropeptide. $^{22}$ It plays an important role in regulating feeding behavior, anti-injury, and anti-hyperalgesia. ${ }^{23,24}$ Our previous studies have found that electrical nerve stimulation can increase the secretion of OrexinA in the lateral hypothalamus and attenuate brain damage by inhibiting oxidative stress, inflammation, and apoptosis. Orexin-A can protect neurons from damage and promote the awakening of rats with traumatic brain injury. ${ }^{10,25}$ However, the effect of orexin-A on NSC lacks sufficient effort. Therefore, we examined the potential of orexin-A to decrease the LPS-stimulated inflammatory responses and signaling pathway of NSCs in this study.

\section{Method}

\section{Materials}

Escherichia coli LPS (O55:B5) was purchased from Solarbio (L8880, China, Beijing). Orexin-A and
SB334867 were purchased in MedchemExpress company. Both western-blot and immunofluorescence primary antibodies were purchased from Cell Signaling Technology Company, and the secondary antibodies were all from Zhongshan Jinqiao Company.

\section{Cell Culture}

NE-4C cells were kindly provided by Stem Cell Bank, Chinese Academy of Science. (SCSP-1501). ${ }^{26}$ NE-4C cells were grown in minimal essential medium (11090081, Gibco) supplemented with 10\% fetal bovine serum (12484028, Gibco), 1\% Glutamax (35050061, Gibco), $1 \%$ Non-essential Amino Acids, 100× (11140050, Gibco), 1\% Sodium Pyruvate $100 \mathrm{mM}$ Solution (11360070, Gibco). Cells were cultured according to ATCC culture method guidelines and maintained in an incubator at $37{ }^{\circ} \mathrm{C}, 5 \% \mathrm{CO} 2$, and $95 \%$ humidity. Cells were plated on $15 \mathrm{ug} / \mathrm{mL}$ poly-L-lysine (Cat\#P-9155, Sigma)-coated culture plates. The cell passage uses $0.05 \%$ trypsin digestion for 5-10 minutes, the growth medium was changed 3 times per week.

\section{Cell Proliferation Assay}

The cell proliferation was assayed using a tetrazolium salt colorimetric assay using PreMix WST-1 according to the manufacturer's instructions (Beyotime, China, Shanghai). The cells were seeded at a density of $5 \times 10^{3}$ cells per well in 96-well plates in a volume of 100ul, and they were incubated for the indicated periods. PreMix WST-1 was added as indicated and incubated for an additional $2 \mathrm{~h}$, and then the absorbance was measured at $450 \pm 20 \mathrm{~nm}$, with 5 replicates per group.

\section{Nitric Oxide (NO) Assay}

Nitric oxide (NO) production was estimated from nitrite contents that were detected using NO assay kits with the Griess reaction (Beyotime, China, Shanghai) according to the manufacturer's instructions. NE-4C cells were plated into 24-well plates and subjected to various treatments. The culture supernatants were obtained 12h, 24h, 36h, $48 \mathrm{~h}$, and $72 \mathrm{~h}$ after treating. A $50 \mathrm{ul}$ sample of the culture supernatant was aliquoted into each well of a 96 well plate, 50ul Griess reagent I (part I: 1\% sulphanilamide) was subsequently added and incubated at room temperature for 3-5 minutes. 50uL of Griess reagent II (part II: $0.1 \%$ naphthyl ethylene diamide dihydrochloride and $2 \%$ phosphoric acid) was added and the pellets were mixed for 
$15 \mathrm{~min}$ and then the absorbance was measured at $540 \pm 20$ $\mathrm{nm}$, with 3 replicates per group.

\section{Scratch Tests Assay}

The scratch test is used to test the migration behavior of NE-4C cells cultured under different conditions. In detail, We rowed three straight lines on the back of the 6-well plate with $0.5-1 \mathrm{~cm}$ intervals. Cells at a density of $5 \times 10^{5}$ / well were seeded into 6-well plates for $24 \mathrm{~h}$. Subsequently, The scratch was made with a $200 \mathrm{ul}$ pipetting spear perpendicular to the three baselines. Then, wash the cells twice with PBS. At last, add $2.5 \mathrm{~mL}$ of serum-free drug medium to continue the culture. We observe cell growth at 0,12 , and 24 hours respectively.

\section{Transwell Migration Assay}

Besides the scratch test, the migration ability of NE-4C cells was also tested by transwell chambers (24-well culture plate, 8-um pore size). NE-4C cells were suspended in a serumfree medium and adjusted to a density of $2 \times 10^{5}$ cells $/ \mathrm{mL}$. 200ul cell suspension was added to the upper chamber of the migration well. On the contrary, a 20\% serum medium was loaded into the lower chamber. Following $24 \mathrm{~h}$ of incubation, cells in the upper chamber were removed using a cotton swab, and the cells that had migrated through to the underside of the insert membranes were fixed with $4 \%$ paraformaldehyde for $20 \mathrm{~min}$ and stained with crystal violet (Solarbio, Shanghai, China) for $20 \mathrm{~min}$.

\section{Hematoxylin-Eosin (HE) Staining}

HE staining was used to observe morphology after fixation of cultured NE-4Ccells. The covered slides with cells were washed thrice with phosphate-buffered saline (PBS), fixed in 4\% paraformaldehyde for $20 \mathrm{~min}$. The slides were stained with hematoxylin dye for 3 minutes, then rinsed with running water, stained with differentiation solution for 30 $\mathrm{s}$, then rinsed with running water, finally stained with eosin dye for 1 minute, and then rinsed with running water.

\section{RNA Extraction and Real-Time Quantitative RT-PCR (qRT-PCR) Analysis}

At $24 \mathrm{~h}$ after treatment, the total RNA was extracted from the NE-4C cells by using the TRizol reagent (Invitrogen). cDNA was synthesized using the Takara PrimeScript RT Reagent Kit (Takara). For qRT-PCR, 800ng total RNA was used in the Takara PrimeScript RT reagent Kit (Takara) following the manufacturer's instructions, and PCR was performed in the CFX96 Real-Time PCR Detection System. The primer sequence is shown in Table 1.

\section{Total Protein Extraction and Western Blotting}

To determine NF-kB and mitogen-activated protein kinases (MAPK) signaling ofNE-4C cells, total proteins were extracted from NE-4C treated with orexinA, SB334867 and LPS using RIPA commercial kits (Beyotime, China, Shanghai). Protein was quantified using a Pierce BCA Protein Assay Kit (Thermo Fisher Scientific), followed by Western blot analysis. Once transferred to a nitrocellulose (NC) filter membrane, antibodies targeting $\beta$-actin (Proteintech, 66009-1, 1:10000), OXR1 (Proteintech, 13514-1, 1:10000), IKB $\alpha$ (CST, 4812, 1:1000), P-IKB $\alpha$ (CST, 2859, 1:1000), P65 (CST, 8242, 1:1000), P-P65 (CST, 3033, 1:1000), Erk (CST, 4695, 1:1000), P-Erk (CST, 4370, 1:1000), P38 (CST, 8690, 1:1000), P-P38 (CST, 4511, 1:1000), JNK (CST, 9252, 1:1000) and P-JNK (CST, 4668, 1:1000) were used with a secondary antibody horseradish peroxidase-labeled goat anti-rabbit or goat anti-mouse ( $1: 10000 ; 1.5 \mathrm{~h}$ of incubation at room temperature). $\beta$-actin

Table I PCR Primers Used in This Study

\begin{tabular}{|l|l|l|}
\hline Gene Target & Primer Sequence & $\mathbf{T m}^{\left.{ }^{\circ} \mathbf{C}\right)}$ \\
\hline IL-I $\beta$ & F 5'-ACTCATTGTGGCTGTGGAGA-3' & 54.5 \\
& R 5'-AGCCTGTAGTGCAGTTGTCT-3' & 54.53 \\
\hline IL6 & F 5'-ACTTCACAAGTCCGGAGAGG-3' & 54.65 \\
\hline TNF $\alpha$ & R 5'-TGCAAGTGCATCATCGTTGT-3' & 54.44 \\
\hline GAPDH & F 5'-CTCATGCACCACCATCAAGG-3' & 54.56 \\
& R 5'-ACCTGACCACTCTCCCTTTG-3' & 54.49 \\
\hline
\end{tabular}


served as an internal control. Protein bands were visualized by enhanced chemiluminescence.

\section{Statistical Analysis}

The SPSS 24 software (IBM, Armonk, NY, USA) was used for statistical analysis. The data are presented as mean \pm standard deviation (SD). Multiple comparisons among all groups were performed by one-way analysis of variance (ANOVA) followed by a post-hoc test. Comparisons between the two groups were performed by the Student's $t$-test. For repeatedly measured outcomes, repeated-measures analysis of variance was used. $\quad \mathrm{P}<0.05$ was considered to be statistically significant.

\section{Results}

\section{Orexin-A Enhanced the Ability of Proliferation and Migration of NE-4C in vitro}

To determine the optimal concentration of Orexin-A that induces the proliferation of NE-4C cells, $10^{-4}, 10^{-3}, 10^{-2}$, 0.1 , and $1 \mathrm{uM}$ Orexin-A was used to treat NE-4C cells for 24 hours, and then the absorbance was measured at $450 \pm 20$ nm with CCK8 kit. As shown in Figures 1A, 0.1uM is the optimal concentration of orexin-A to induce NE-4C proliferation $(\mathrm{P}=0.001)$. Subsequently, we used SB-334867, which is an excellent selective blood-brain barrier permeability orexin-A (OXR1) receptor antagonist (Figure 1B). The results showed that the difference in the Orexin-A +SB334867 group was not statistically significant compared with the control group.

Horizontal migration ability was assessed using a cell scratch assay. The figure (Figure 1C and D) showed that the Orexin-A treatment group can promote the healing of NE-4C cells at $12 \mathrm{~h}$ and $24 \mathrm{~h}$ after scratching compared to the control group. Although the orexin-A+SB334867 group has a statistically significant difference compared with the control group at 24 hours. However, the orexin-A+SB334867 group had poor cell healing ability after scratches. Subsequently, The transwell migration assay is used to evaluate vertical migration ability. The results (Figure $1 \mathrm{E}$ and $\mathrm{F}$ ) illustrated that orexin-A increased NE-4C migration numbers than the control group. The results revealed that orexin-A raised NE-4C migration.

\section{Orexin-A Relieves LPS-Induced Inflammatory Injury}

CCK8 is used to evaluate the effect of LPS on NE-4C cell viability. As shown in Figure 2A, LPS treatment alone, with increasing time, the cell viability gradually decreased. The cell viability of the Orexin-A+LPS group was higher than that of the LPS alone group. Therefore, we inferred that orexin-A can reduce the inflammatory damage caused by LPS. Again, we also evaluated the release of NO with the Griess kit. To our surprise, the NO release in the Orexin-A group was higher than that in the LPS alone group (Figure 2B). Therefore, we speculate that Orexin-A can promote the release of $\mathrm{NO}$ from NE-4C cells and act on the central and peripheral nervous systems. HE staining showed that the cells were spindle-shaped or irregular, with little cytoplasm (Figure 2C). As the duration of LPS increases, the changes in cell morphology become more obvious (Figure 2C).

qRT-PCR is used to detect the expression of inflammatory factors. It was found that Orexin-A can reduce the expression of related inflammatory factors. Moreover, when the concentration of Orexin-A was $10^{-1} \mathrm{uM}$, the expression of inflammatory factors IL- 6 and TNF- $\alpha$ was the lowest, while the expression of IL-1 $\beta$ was the lowest when the concentration of Orexin-A was $10^{-2} \mathrm{uM}$ (Figure 2D).

\section{Orexin-A Inhibits LPS-Induced NF-KB Signal Activation}

We investigated changes in the protein levels of LPSinduced signaling pathways by Western blot (Figure 3A) and quantified the data (Figure 3B-D). As seen in Figure 3, the $\mathrm{p}-\mathrm{IkB} \alpha$ was downregulated as $\mathrm{IkB} \alpha$ proteins were upregulated by LPS significantly compared with control in NSCs $(p<0.05)$, as p-p65 were increased compared with total P65 by LPS in NSCs. In contrast, Orexin-A recovered $\mathrm{IkB} \alpha$ and attenuated $\mathrm{p}$-IkB $\alpha$ and $\mathrm{p}$-p65 expression to LPSinduced NSCs $(\mathrm{p}<0.05)$. After adding SB334867, the result is the opposite. Therefore, we conclude that Orexin-A may inhibit LPS-induced NF-KB signal activation.

\section{Orexin-A Decreases LPS-Induced Phosphorylation of MAPK/P38/Erk (I/2) Pathway}

On the other hand, we also verified the related proteins of the MAPK signaling pathway. As shown in Figure 4B-H, all nonphosphorylation pathways of MAPKs cannot be significantly 


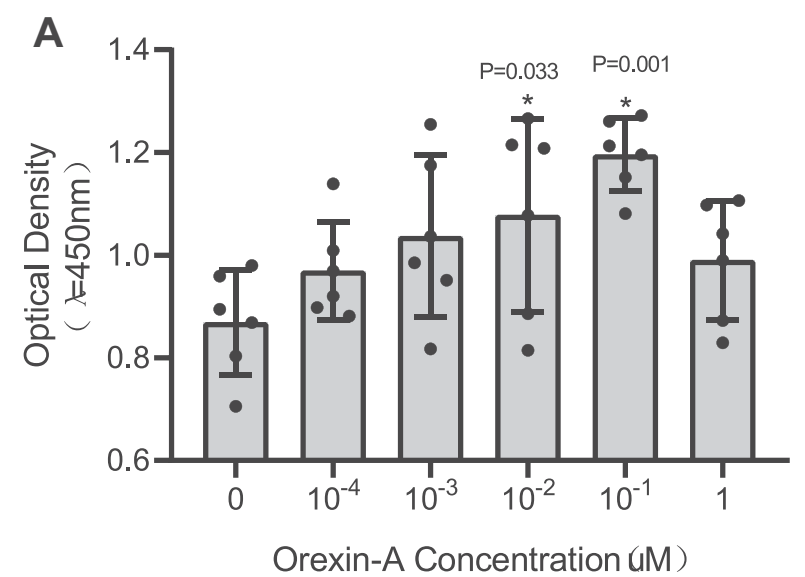

B

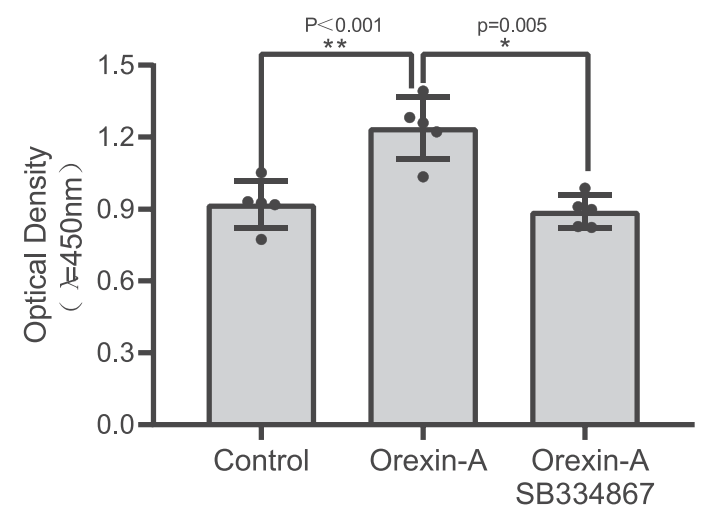

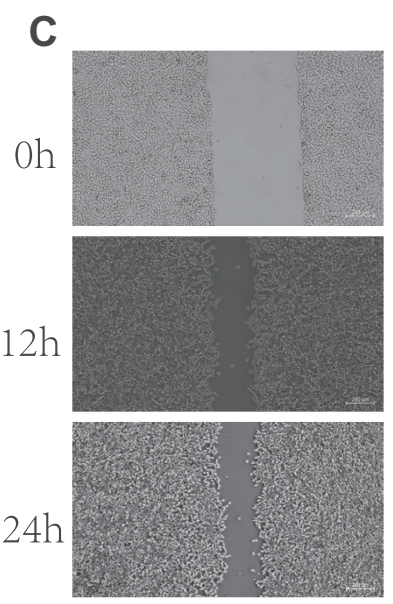

Control
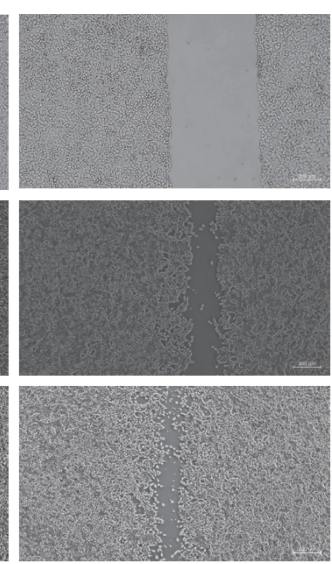

Orexin-A

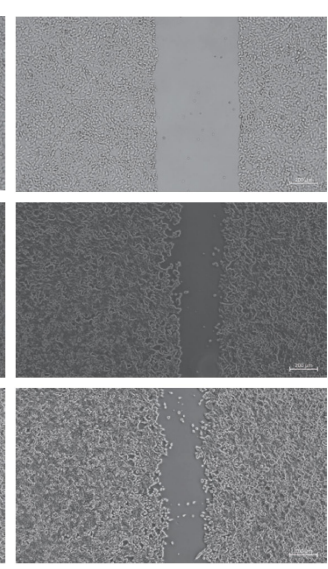

Orexin-A SB334867
D

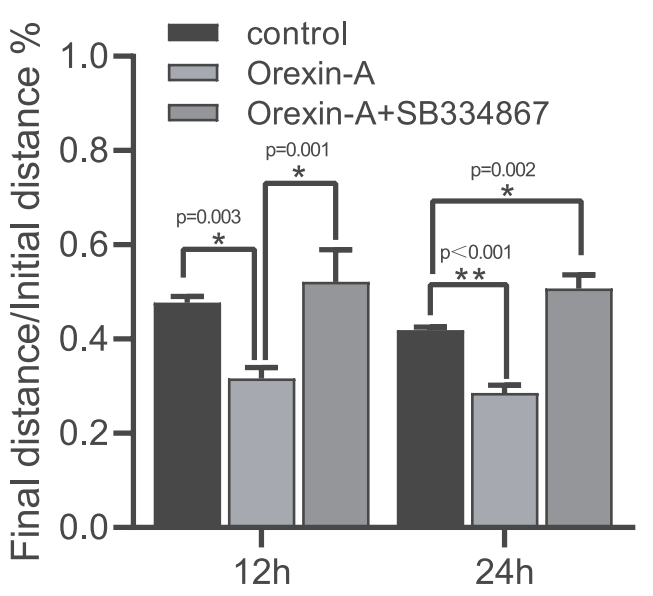

E

$\mathbf{F}$

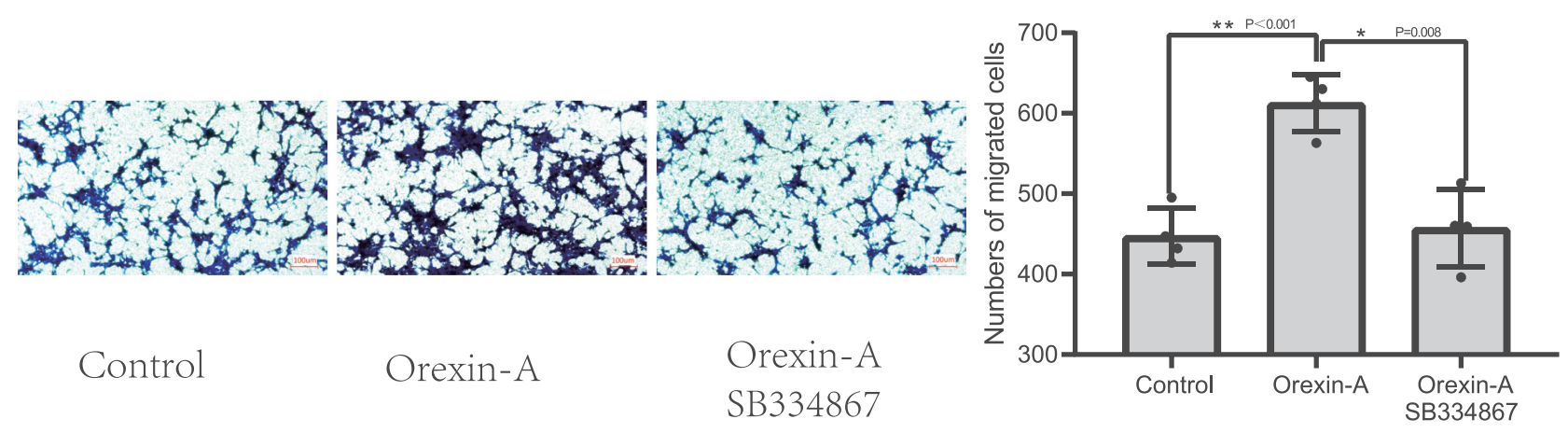

Figure I Orexin-A enhanced the ability of proliferation and migration of NE-4C in vitro. (A) Effects of Orexin-A at Different Concentrations on Proliferation of Neural Stem Cells $(F=3.889, P=0.008)$. (B) The effect of SB334867 on the proliferation of neural stem cells $(F=17.942, P<0.00 I)$. (C and $D)$ Cell scratch test $(I 2 h$ : $F=19.907$, $\mathrm{P}=0.002$; 24h: $\mathrm{F}=95.64, \mathrm{P}<0.00 \mathrm{I})$. ( $\mathrm{E}$ and $\mathrm{F})$ Cell migration experiment $(\mathrm{F}=2 \mathrm{I} .678, \mathrm{P}<0.00 \mathrm{I})$. Figure legend indicates the $\mathrm{P}$-value for $A N O V A$, and the $\mathrm{P}$-value for post-hoc test is noted on the graph. The value were presented as the mean \pm SDM of three or more independent experiments. ${ }^{*} \mathrm{p}<0.05$ and $* * p<0.00 \mathrm{I}$ means the difference is statistically significant.

regulated by LPS or Orexin-A. Remarkably, the evocation of c-Jun N-terminal kinase (JNK) signaling was not significantly influenced by Orexin-A; nevertheless, the evocation of $\mathrm{p} 38$ -
MAPK and ERK signaling changed. In brief, Orexin-A decreases LPS-induced phosphorylation of MAPK/P38/ Erk (1/2) signaling pathways. 
A

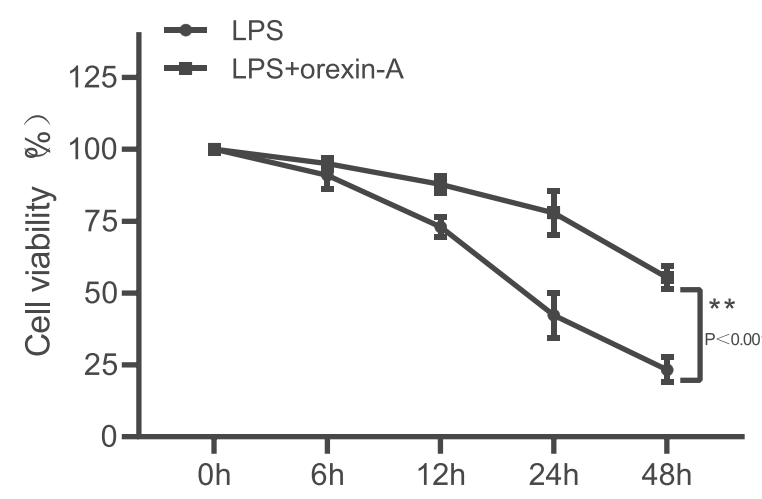

B

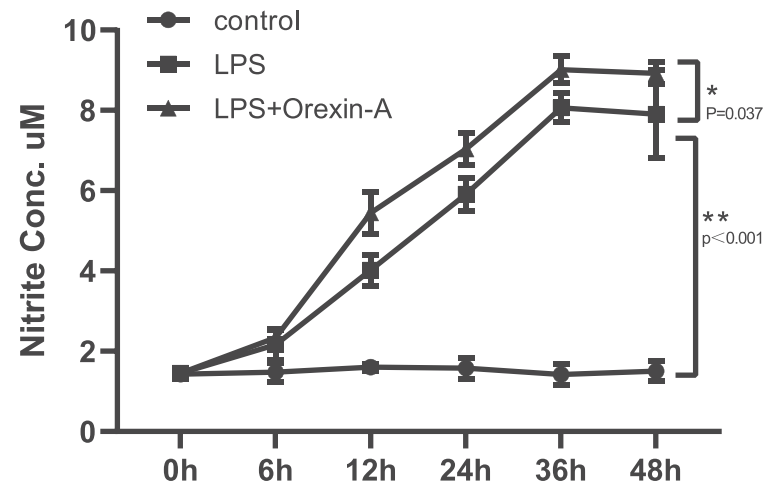

C
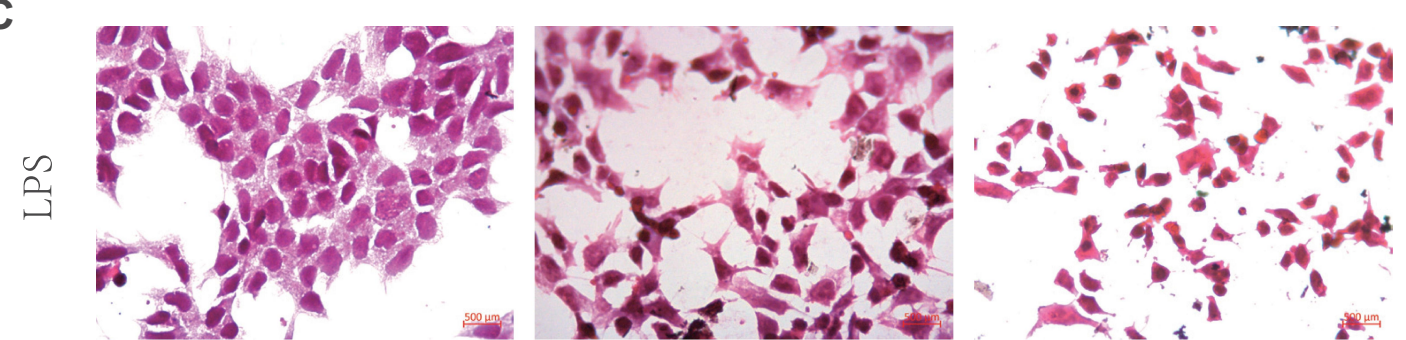

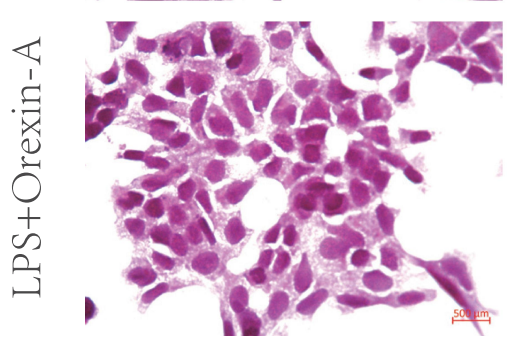

D

Oh

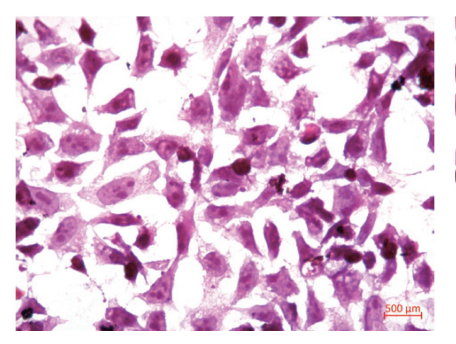

$24 \mathrm{~h}$

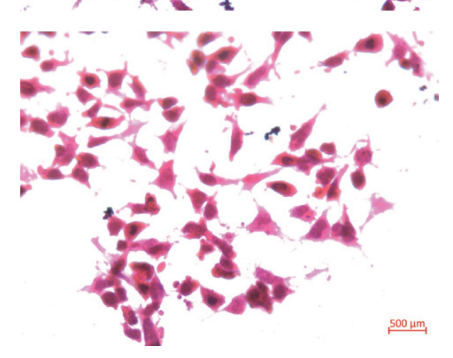

$48 \mathrm{~h}$

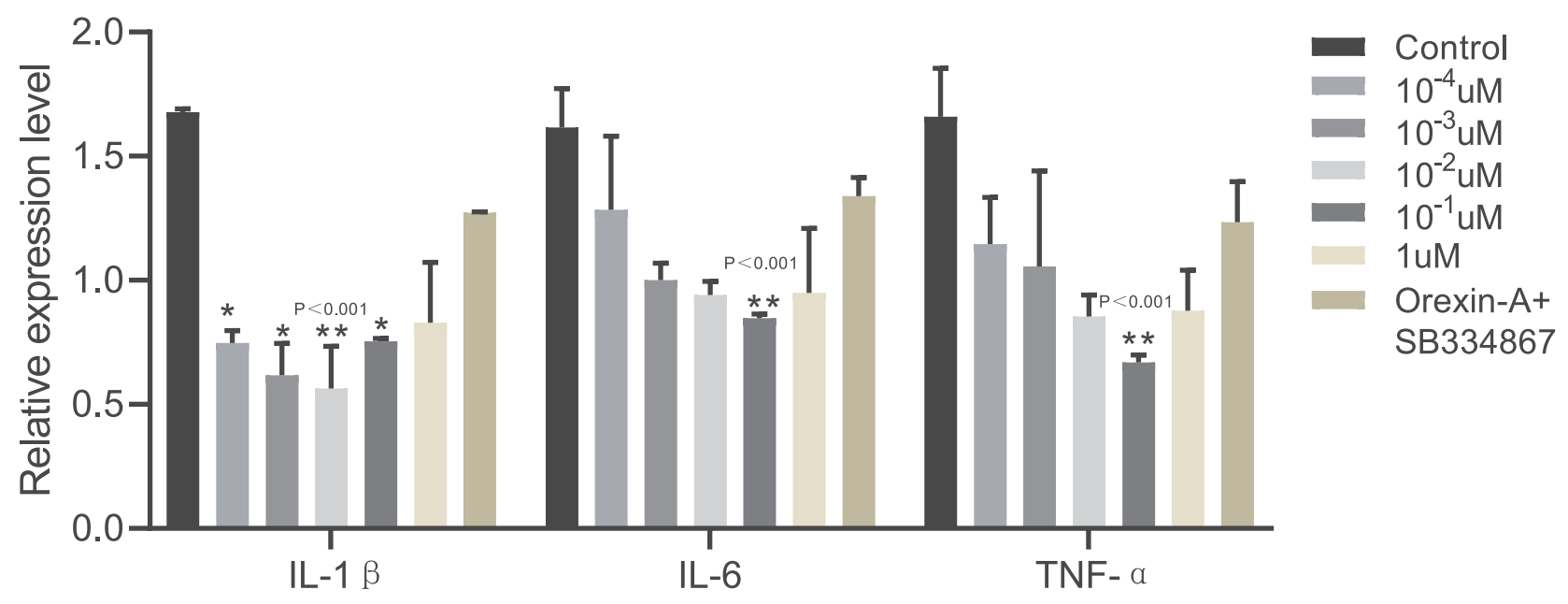

Figure 2 Orexin-A relieves LPS-induced inflammatory injury. (A) Cell viability after LPS treatment ( $F=30.536, P<0.00 \mathrm{I})$. (B) NO production determined using a Griess reagent assay kit on cells treated with LPS and Orexin-A for the indicated times ( $F=65.566, P<0.00 \mathrm{I})$. (C) HE staining to observe the morphology of NSCs induced by orexin-A treatment of LPS. (D) The pro-inflammatory cytokines including IL-I $\beta$ ( $F=31.73, P<0.00 \mathrm{I}), \mathrm{IL}-6 \quad(\mathrm{~F}=8.452, \mathrm{P}=0.00 \mathrm{I})$ and $\mathrm{TNF}-\alpha(\mathrm{F}=7.729, \mathrm{P}=0.00 \mathrm{I})$ were determined by PCR in NSCs. Figure legend indicates the p-value for ANOVA, and the p-value for post-hoc test is noted on the graph. The value were presented as the mean \pm SDM of three or more independent experiments. ${ }^{*} p<0.05$ and $*^{*} p<0.001$ means the difference is statistically significant. 
A

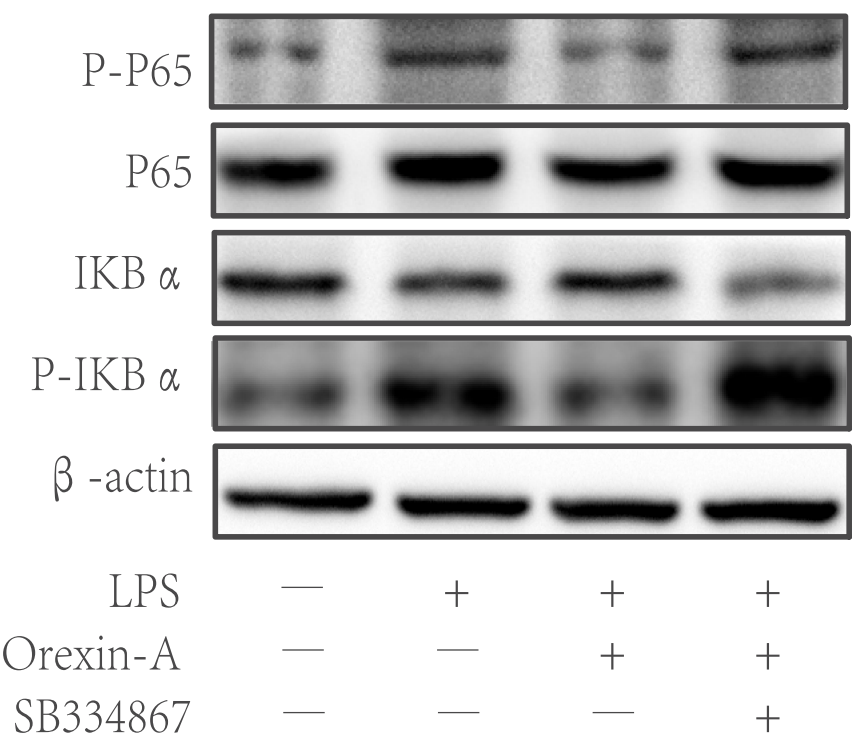

B

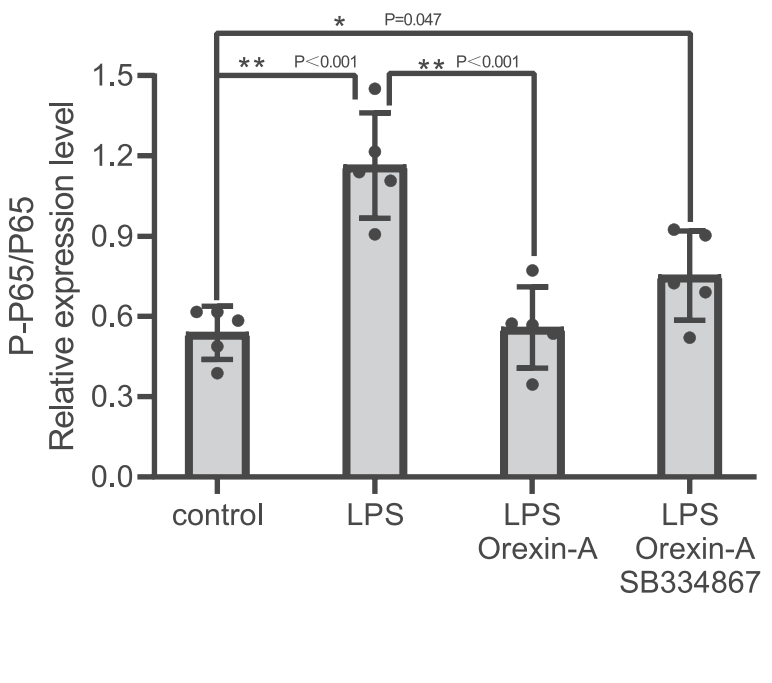

D

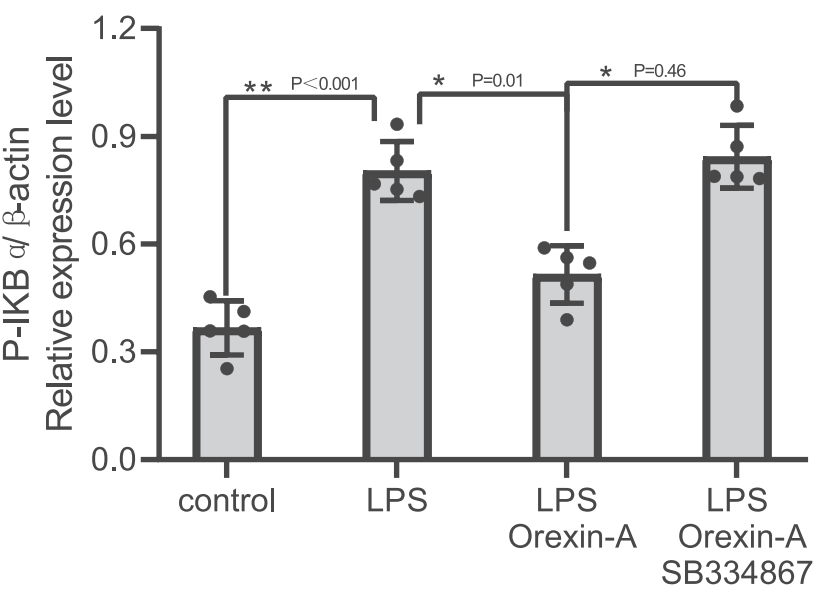

Figure 3 Orexin-A inhibits LPS-induced NF-kB signal activation. NSCs without Orexin-A or LPS treatment were considered as control. (A) Western blotting result; (B-D) gray analysis of Western blotting. The NF-kB signaling proteins of NSCs including, IKB $\alpha(F=29.166, P<0.00 I), P-I K B \alpha(F=39.948, P<0.00 I), P-p 65 / p 65(F=I 6.997, P<0.00 I)$ were determined by Western blotting. Figure legend indicates the $\mathrm{p}$-value for ANOVA, and the $\mathrm{p}$-value for post-hoc test is noted on the graph. The value were presented as the mean \pm SDM of three or more independent experiments. ${ }^{*} \mathrm{p}<0.05$ and ${ }^{* *} \mathrm{p}<0.001$ means the difference is statistically significant.

\section{Discussion}

NSC is a cell with division potential and self-renewal ability. It can not only repair and replace neurons, but also secrete neurotrophic factors and immunomodulate the neural microenvironment. ${ }^{27}$ Stem cell transplantation is becoming more and more popular, but what cannot be ignored is the interaction between the transplanted NSC and the damaged host immune cells. ${ }^{28}$ How to put it into clinical application is the most concerned issue of the entire neuroscience community. Our study found that orexin-A can mediate NF-KB and MAPK signal pathways to inhibit the inflammatory response of neural stem cells. Orexin-A combined with neural stem cell transplantation can reduce the damage of the host immune response. It provides a new idea for the immune response of neural stem cell transplantation. 
A
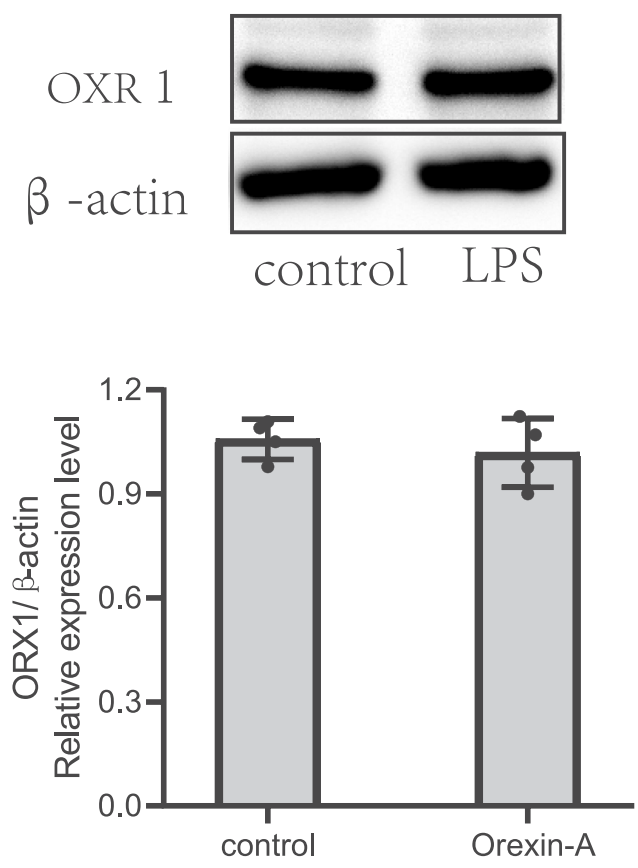

B

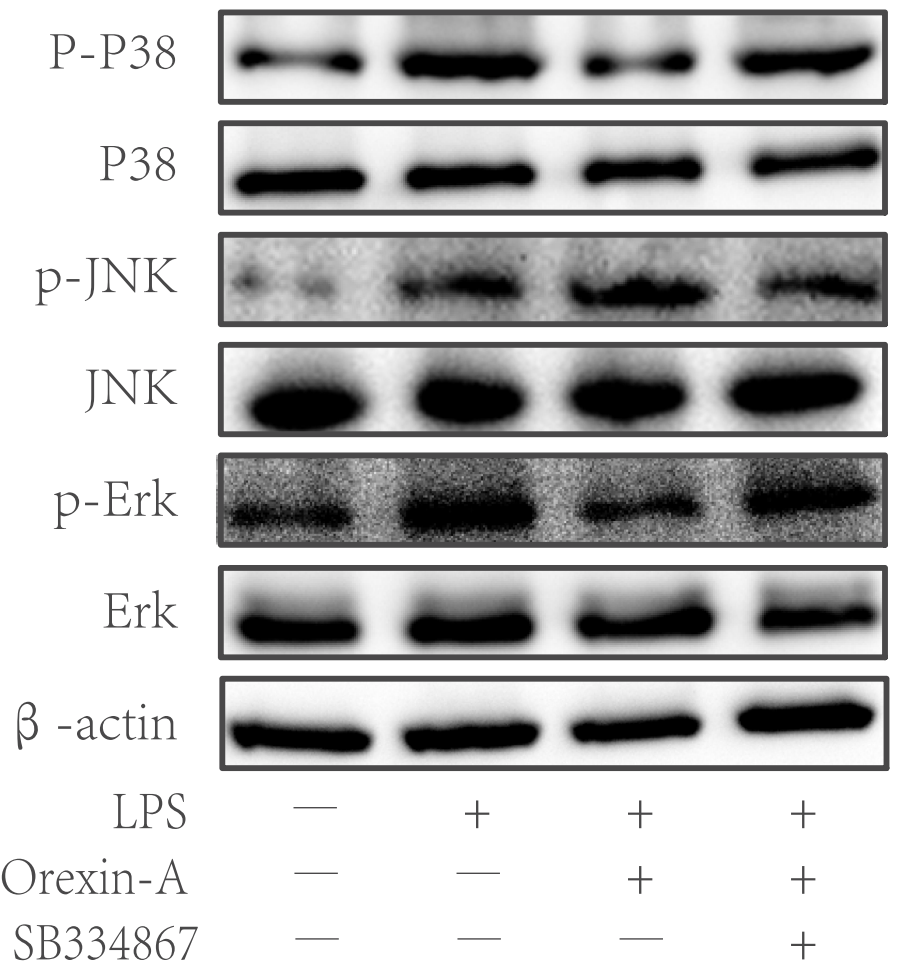

E
C

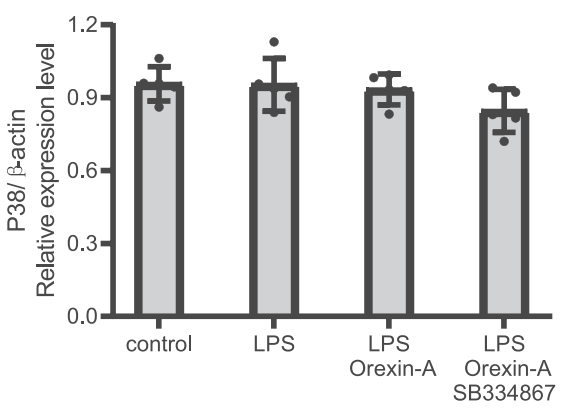

F

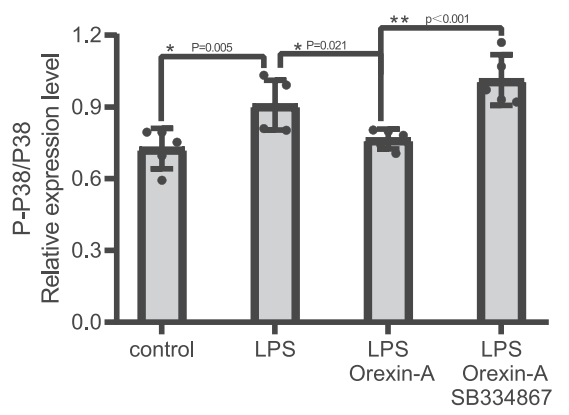

D

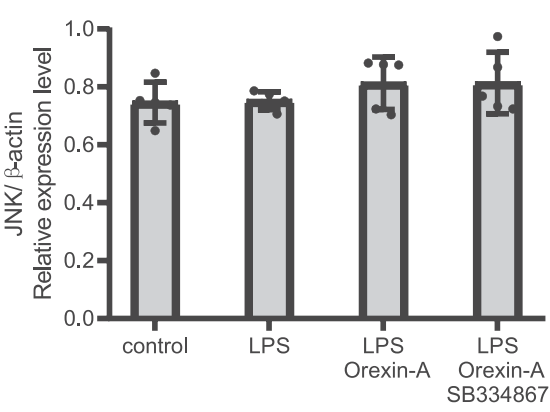

G

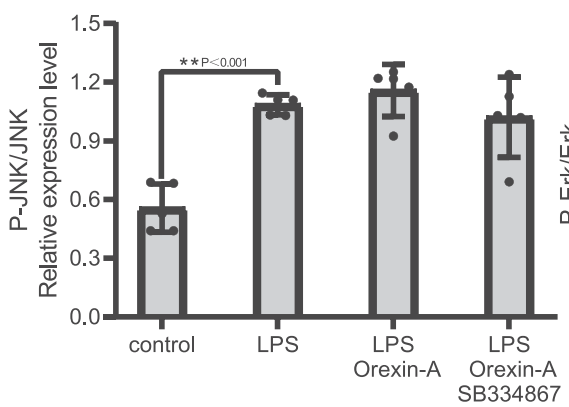

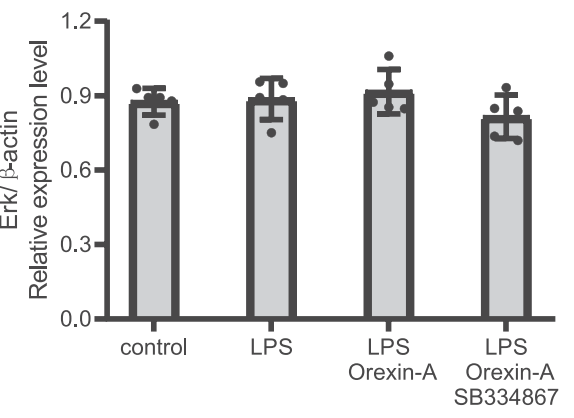

H

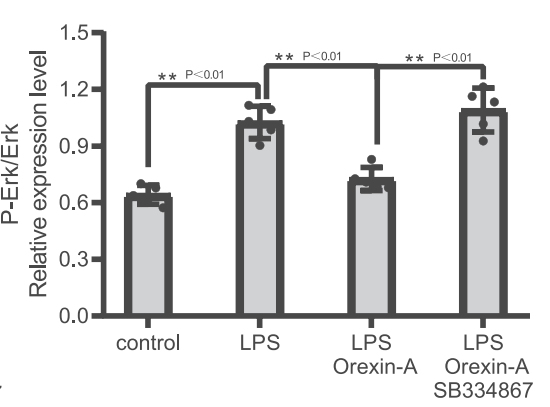

Figure 4 Orexin-A decreases LPS-induced phosphorylation of MAPK/P38/Erk (I/2) pathway. (A) Expression of OXRI in Orexin-A treatment group and control group. (BH) The MAPK signaling proteins of NSCs including, $\mathrm{P}-\mathrm{P} 38 / \mathrm{P} 38(\mathrm{~F}=\mathrm{II} .256, \mathrm{P}<0.00 \mathrm{I})$, $\mathrm{P}$-Erk/Erk $(\mathrm{F}=35.946, \mathrm{P}<0.00 \mathrm{I}), \mathrm{P}-\mathrm{JNK} / \mathrm{JNk}(\mathrm{F}=19.00 \mathrm{I}, \mathrm{P}<0.00 \mathrm{I})$, and were determined by Western blotting. Figure legend indicates the $\mathrm{p}$-value for ANOVA, and the $\mathrm{p}$-value for post-hoc test is noted on the graph. The value were presented as the mean \pm SDM of three or more independent experiments. ${ }^{*} p<0.05$ and ${ }^{*} *_{p}<0.001$ means the difference is statistically significant. 


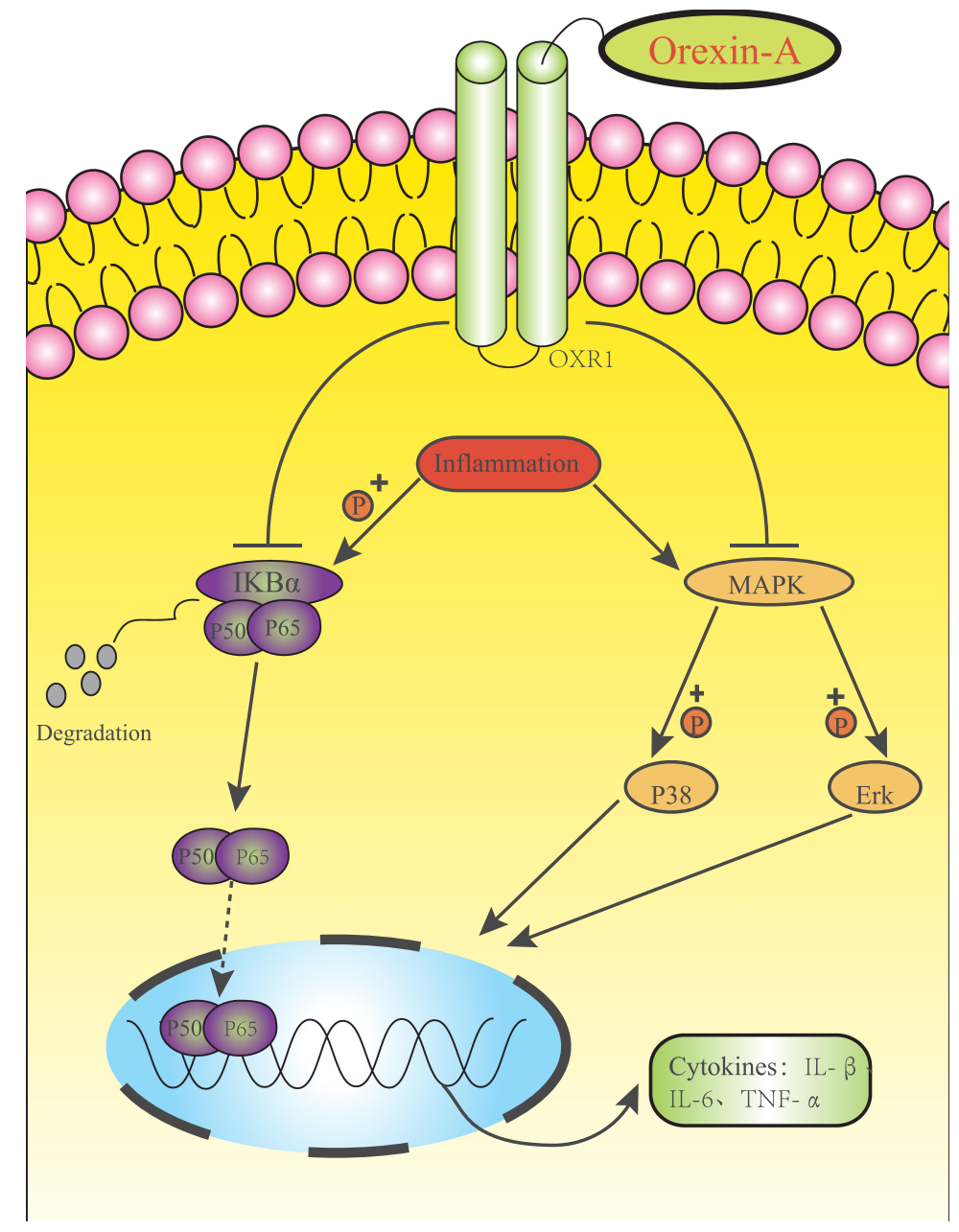

Figure 5 Schematic diagram of the potential mechanism and protective effect of orexin-A on NE-4C inflammatory injury. Orexin-A attenuates inflammatory responses in lipopolysaccharide-induced neural stem cells by regulating NF-kB and phosphorylation of MAPK/P38/Erk pathways, which protects NSC transplantation versus host response.

Although there has been an agreement that the functions of Orexin-A differ in the digestive system and nervous system, conflict observations were reported. N. Messal's research shows that Ectopic expression of OXR1 in ulcerative colitis mediates the antiinflammatory effect of Orexin-A. ${ }^{29}$ Another study showed that Orexin-A can promote the proliferation and migration of cancer stem cells. ${ }^{30}$ The other result reversely suggested that the proliferation of neural progenitor cells derived from rat fetal brains was not affected by Orexin-A treatment in vitro, and the orexin receptor 1 (OXR1) protein was not expressed in these cells. ${ }^{31}$ That study may have been underpowered. The reason is that N. ITO's research used the adult mouse brain extract as a positive control, and the result found that neural precursor cells did not express the OXR1 receptor. Surprisingly, Our study found that both the untreated group of NE-4C cells and the Orexin-A treated group had OXR1 receptor expression (Figure 4A). Our research shows that Orexin-A can promote the proliferation and migration of NSC cells, which is beneficial to repair the neural circuit after NSC transplantation.

Our research shows that Orexin-A decreases LPSinduced phosphorylation of the MAPK/P38/Erk (1/2) pathway. This result is consistent with recent research. For example, Orexin-A-induced osteoblastic formation and matrix mineralization and the activation of the PKD/p38 MAPK pathway are mediated by OXR $1 .{ }^{32}$ Orexin-A could stimulate the expression of GLUT4 in a glucose-dependent manner in primary hepatocytes via Erk1/2, JNK, and p38 signaling. ${ }^{33}$ In 
addition, Dandan's studies have shown that Orexin-A exerted its neuroprotective effects in vivo and in vitro by suppressing over-activated autophagy by modulating the OXR1mediated MAPK/Erk/mTOR pathway. ${ }^{34}$

Several limitations are worthy of discussion. At first, These conclusions were based on the responses of NE-4C cell lines and so might not reflect processes in the intact body. We are now confirming our results in Primary cells and mice. In addition, Our study did not clarify the specific connection between the NF-kB signaling pathway and the MAPK/p38/Erk signaling pathway. We will continue to discuss whether they are front-to-back or side by side. This study also owns some shining points or highlights: (1) It is the first time that Orexin-A can promote cell proliferation and migration at the level of neural stem cells. (2) Orexin-A can facilitate protecting the inflammatory responses of NSCs, and provide a beneficial function for the application of NSCs in the therapy.

\section{Conclusion}

Our results uncover the role of Orexin-A in the regulation of neural stem cells. We have demonstrated that Orexin-A attenuates inflammatory responses in lipopolysaccharideinduced neural stem cells by regulating NF-kB and phosphorylation of MAPK/P38/Erk pathways (Figure 5). Our research results show that Orexin-A is expected to become a promising new therapeutic target in neural stem cell transplantation.

\section{Statement of Ethics}

The research object of this paper is a cell line, no authorization from an ethics research committee was required.

\section{Funding}

This study was funded by the National Natural Science Foundation of China (Grant No. 81860409 and No. 81660382) and Graduate Students Innovation Fund Project in Jiangxi Province (No. YC2020-B034).

\section{Disclosure}

The authors report no conflicts of interest in this work.

\section{References}

1. Zhang M, Xu L, Yang H. Schisandra chinensis fructus and its active ingredients as promising resources for the treatment of neurological diseases. Int J Mol Sci. 2018;19(7).
2. Ye J, Yao J-P, Wang XU, et al. Neuroprotective effects of ginsenosides on neural progenitor cells against oxidative injury. Mol Med Rep. 2016;13(4):3083-3091. doi:10.3892/mmr.2016.4914

3. Huang Y, Wan C, Wu G. Acute kidney injury after a stroke: a PRISMA-compliant meta-analysis. Brain Behav. 2020;10(9): e01722. doi:10.1002/brb3.1722

4. Liao LY, Lau B-M, Sánchez-Vidaña D, et al. Exogenous neural stem cell transplantation for cerebral ischemia. Neural Regen Res. 2019;14 (7):1129-1137. doi:10.4103/1673-5374.251188

5. Haghi M, Masoudi R, Najibi SM. Distinctive alteration in the expression of autophagy genes in Drosophila models of amyloidopathy and tauopathy. Ups J Med Sci. 2020;125(4):265-273. doi:10.1080/ 03009734.2020.1785063

6. Tejeda GS, Esteban-Ortega GM, San Antonio E, et al. Prevention of excitotoxicity-induced processing of BDNF receptor TrkB-FL leads to stroke neuroprotection. EMBO Mol Med. 2019;11(7):e9950. doi:10.15252/emmm.201809950

7. Birbeck GL, Meyer AC, Ogunniyi A. Nervous system disorders across the life course in resource-limited settings. Nature. 2015;527 (7578):S167-71. doi:10.1038/nature16031

8. Platschek S, Cuntz H, Vuksic M, et al. A general homeostatic principle following lesion induced dendritic remodeling. Acta Neuropathol Commun. 2016;4:19. doi:10.1186/s40478-016-0285-8

9. Feng Y, Jiang C, Yang F, et al. Apocynum venetum leaf extract protects against $\mathrm{H}(2) \mathrm{O}(2)$-induced oxidative stress by increasing autophagy in PC12 cells. Biomed Rep. 2020;13(2):6. doi:10.3892/ br.2020.1313

10. Tang Y, Dong X, Chen G, et al. Vagus nerve stimulation attenuates early traumatic brain injury by regulating the NF- $\mathrm{KB} / \mathrm{NLRP} 3$ signaling pathway. Neurorehabil Neural Repair. 2020;34(9):831-843. doi: $10.1177 / 1545968320948065$

11. Zamboni M, Llorens-Bobadilla E, Magnusson JP, et al. A widespread neurogenic potential of neocortical astrocytes is induced by injury. Cell Stem Cell. 2020;27(4):605-617.e5. doi:10.1016/j.stem.20 20.07.006

12. Griffin TA, Anderson HC, Wolfe JH. Ex vivo gene therapy using patient iPSC-derived NSCs reverses pathology in the brain of a homologous mouse model. Stem Cell Rep. 2015;4(5):835-846. doi:10.1016/j.stemcr.2015.02.022

13. Robinson AP, Foraker JE, Ylostalo J, et al. Human stem/progenitor cells from bone marrow enhance glial differentiation of rat neural stem cells: a role for transforming growth factor $\beta$ and Notch signaling. Stem Cells Dev. 2011;20(2):289-300. doi:10.1089/scd.2009.0444

14. Di Lorenzo F, Pither MD, Martufi M, et al. Pairing bacteroides vulgatus LPS structure with its immunomodulatory effects on human cellular models. ACS Cent Sci. 2020;6(9):1602-1616. doi:10.1021/acscentsci.0c00791

15. Zhu W, Li J, Liu Y, et al. Mesencephalic astrocyte-derived neurotrophic factor attenuates inflammatory responses in lipopolysaccharide-induced neural stem cells by regulating NF- $\mathrm{kB}$ and phosphorylation of $\mathrm{p} 38$ MAPKs pathways. Immunopharmacol Immunotoxicol. 2016;38 (3):205-213. doi:10.3109/08923973.2016.1168433

16. Ni J, Zhao Y, Su J, et al. Toddalolactone protects lipopolysaccharideinduced sepsis and attenuates lipopolysaccharide-induced inflammatory response by modulating HMGB1-NF- $\mathrm{KB}$ translocation. Front Pharmacol. 2020;11:109. doi:10.3389/fphar.2020.00109

17. He P, Gelissen IC, Ammit AJ. Regulation of ATP binding cassette transporter A1 (ABCA1) expression: cholesterol-dependent and - independent signaling pathways with relevance to inflammatory lung disease. Respir Res. 2020;21(1):250. doi:10.1186/s12931-020-01515-9

18. Zheng C, Yin Q, Wu H. Structural studies of NF-kB signaling. Cell Res. 2011;21(1):183-195. doi:10.1038/cr.2010.171

19. Tang X, Huang B, Lin S, et al. CgMyD88s serves as an innate immune system plug during ostreid herpesvirus 1 infection in the Pacific Oyster (Crassostrea gigas). Front Immunol. 2020;11:1247. doi:10.3389/fimmu.2020.01247 
20. Georg I, Díaz-Barreiro A, Morell M, et al. BANK1 interacts with TRAF6 and MyD88 in innate immune signaling in B cells. Cell Mol Immunol. 2020;17(9):954-965. doi:10.1038/s41423-019-0254-9

21. Malireddi RKS, Gurung P, Kesavardhana S, et al. Innate immune priming in the absence of TAK1 drives RIPK1 kinase activity-independent pyroptosis, apoptosis, necroptosis, and inflammatory disease. J Exp Med. 2020;217(3). doi:10.1084/jem.20191644.

22. Tsujino N, Sakurai T. Role of orexin in modulating arousal, feeding, and motivation. Front Behav Neurosci. 2013;7:28. doi:10.3389/ fnbeh.2013.00028

23. Zordan MA, Sandrelli F. Circadian clock dysfunction and psychiatric disease: could fruit flies have a say? Front Neurol. 2015;6:80. doi:10.3389/fneur.2015.00080

24. Janockova J, Dolezal R, Nepovimova E, et al. Investigation of new orexin 2 receptor modulators using in silico and in vitro methods. Molecules. 2018;23(11). doi:10.3390/molecules23112926.

25. Dong X, Feng Z. Wake-promoting effects of vagus nerve stimulation after traumatic brain injury: upregulation of orexin-A and orexin receptor type 1 expression in the prefrontal cortex. Neural Regener Res. 2018;13(2):244-251. doi:10.4103/1673-5374.226395

26. Schlett K, Madarász E. Retinoic acid induced neural differentiation in a neuroectodermal cell line immortalized by p53 deficiency. J Neurosci Res. 1997;47(4):405-415. doi:10.1002/(SICI)1097-4547(19970215)47:4<405::AID-JNR6>3.0.CO;2-I

27. Gao M, Dong Q, Zhang H, et al. Syringe needle skull penetration reduces brain injuries and secondary inflammation following intracerebral neural stem cell transplantation. Exp Ther Med. 2017;13 (3):885-890. doi:10.3892/etm.2017.4054

28. Yoon Y, Kim HS, Jeon I, et al. Implantation of the clinical-grade human neural stem cell line, CTX0E03 rescues the behavioral and pathological deficits in the quinolinic acid-lesioned rodent model of Huntington's disease. Stem Cells. 2020;38(8):936-947. doi:10.1002/ stem. 3191
29. Messal N, Fernandez N, Dayot S, et al. Ectopic expression of OX1R in ulcerative colitis mediates anti-inflammatory effect of orexin-A. Biochimica Et Biophysica Acta Mol Basis Dis. 2018;1864 (11):3618-3628. doi:10.1016/j.bbadis.2018.08.023

30. Suo L, Chang X, Zhao Y. The Orexin-A-regulated Akt/mTOR pathway promotes cell proliferation through inhibiting apoptosis in pancreatic cancer cells. Front Endocrinol (Lausanne). 2018;9:647. doi:10.3389/fendo.2018.00647

31. Ito $\mathrm{N}$, Yabe $\mathrm{T}$, Gamo $\mathrm{Y}$, et al. I.c.v. administration of orexin-A induces an antidepressive-like effect through hippocampal cell proliferation. Neuroscience. 2008;157(4):720-732. doi:10.1016/j. neuroscience.2008.09.042

32. Han X, Zhou J, Peng W. Orexins facilitates osteogenic differentiation of MC3T3-E1 cells. IUBMB Life. 2018;70(7):633-641. doi:10.1002/ iub. 1757

33. Zhang C, Sun C, Wang B, et al. Orexin-A stimulates the expression of GLUT4 in a glucose dependent manner in the liver of orange-spotted grouper (Epinephelus coioides). Comp Biochem Physiol Mol Integr Physiol. 2016;199:95-104. doi:10.1016/j. cbpa.2016.05.027

34. $\mathrm{Xu} \mathrm{D}$, Kong $\mathrm{T}$, Zhang $\mathrm{S}$, et al. Orexin-A protects against cerebral ischemia-reperfusion injury by inhibiting excessive autophagy through OX1R-mediated MAPK/ERK/mTOR pathway. Cell Signal. 2021;79:109839. doi:10.1016/j.cellsig.2020.109839
Journal of Inflammation Research

\section{Publish your work in this journal}

The Journal of Inflammation Research is an international, peerreviewed open-access journal that welcomes laboratory and clinical findings on the molecular basis, cell biology and pharmacology of inflammation including original research, reviews, symposium reports, hypothesis formation and commentaries on: acute/chronic inflammation; mediators of inflammation; cellular processes; molecular mechanisms; pharmacology and novel anti-inflammatory drugs; clinical conditions involving inflammation. The manuscript management system is completely online and includes a very quick and fair peerreview system. Visit http://www.dovepress.com/testimonials.php to read real quotes from published authors. 